\title{
Improved integrity constraints checking in distributed databases by exploiting local checking.
}

\begin{abstract}
Most of the previous studies concerning checking the integrity constraints in distributed database derive simplified forms of the initial integrity constraints with the sufficiency property, since the sufficient test is known to be cheaper than the complete test and its initial integrity constraint as it involves less data to be transferred across the network and can always be evaluated at the target site (single site). Their studies are limited as they depend strictly on the assumption that an update operation will be executed at a site where the relation specified in the update operation is located, which is not always true. Hence, the sufficient test, which is proven to be local test by previous study, is no longer appropriate. This paper proposes an approach to checking integrity constraints in a distributed database by utilizing as much as possible the local information stored at the target site. The proposed approach derives support tests as an alternative to the existing complete and sufficient tests proposed by previous researchers with the intention to increase the number of local checking regardless the location of the submitted update operation. Several analyses have been performed to evaluate the proposed approach, and the results show that support tests can benefit the distributed database, where local constraint checking can be achieved.
\end{abstract}

Keyword: Distributed databases; Integrity constraints; Constraints checking; Local checking. 